ORIGINAL ARTICLE

\title{
Is erectile dysfunction a sentinel symptom for cardiovascular autonomic neuropathy in patients with type 2 diabetes?
}

\author{
M. Debono ${ }^{1}$, E. Cachia ${ }^{1}$, A. Cassar ${ }^{2}$, N. Calleja ${ }^{3}$, M. Mallia $^{1}$ \& J. Vassallo ${ }^{1}$ \\ 1 Department of Diabetes and Endocrinology, St Luke's Hospital, Gwardamangia, Malta; \\ 2 Department of Cardiology, St Luke's Hospital, Gwardamangia, Malta; \\ 3 Health Information Department, St Luke's Hospital, Gwardamangia, Malta
}

\section{Keywords}

Cardiovascular autonomic

neuropathy-erectile dysfunction-type 2 diabetes

\section{Correspondence \\ Dr Miguel Debono, Department of Diabetes and Endocrinology, Sheffield Teaching \\ Hospitals, Herries Road, Sheffield, South \\ Yorkshire S5 7AU, UK. \\ Tel.: +44 776019 2117; \\ Fax: +44 114226 6924; \\ E-mail: m.debono@sheffield.ac.uk}

Accepted: July 26, 2007

\begin{abstract}
Summary
The study investigated whether there is a significant association between erectile dysfunction (ED) secondary to autonomic failure, and cardiovascular autonomic neuropathy (CAN) in male patients suffering from type 2 diabetes. Twenty-two patients suffering from type 2 diabetes were recruited for this study after satisfying the stringent exclusion criteria used in the first stage. They had no evidence of overt cardiovascular disease, hypertension, neurological, renal or thyroid disease. Each subject was assessed for ED and CAN using standardized tests. Six patients were suffering from CAN while 10 patients were suffering from ED. There was no significant association between CAN and autonomic $\mathrm{ED}(P=1)$. Three patients with normal erectile function had CAN, whilst three patients with ED had CAN. Further analysis demonstrates a significant increase in association between ED and CAN with age $(P=0.036)$. These results show that ED secondary to autonomic neuropathy is not significantly associated with CAN in this specific group of patients. Nonetheless, the study reveals that $\mathrm{ED}$ is a sentinel symptom for future development of CAN.
\end{abstract}

\section{Introduction}

Diabetic autonomic neuropathy is one of the least recognised and understood complications of diabetes despite its significant negative impact on survival and quality of life in people with diabetes (Vinik et al., 2003). Diabetes may affect any part of the autonomic nervous system harming sweat glands, the gastrointestinal tract system, the adrenal medullary system, and last but not least the cardiovascular and the genitourinary system. The crucial point about diabetic autonomic neuropathy is that subclinically it can occur as early as within a year of diagnosis in patients with type 2 diabetes (Pfeifer et al., 1984). Therefore, mortality and morbidity may be increased at a very early stage in patients with diabetes. In fact the 5 -year mortality rate is five times higher for individuals with cardiovascular autonomic neuropathy (CAN) than for individuals without cardiovascular autonomic involvement (Ziegler, 1999).

Erectile dysfunction (ED) is a common complication of diabetes; the reported prevalence ranges from $27 \%$ to
$75 \%$ (Bacon et al., 2002). ED is defined as the consistent inability to attain and maintain an erection adequate for sexual intercourse, usually qualified by being present for several months and occurring at least half the time. There are multiple causes for ED in patients with diabetes. These include vascular, endocrine, neurological and psychogenic aetiologies. Autonomic neuropathy is one of the main causes for ED (Ziegler, 2001).

Quadri et al. (1989) studied diabetic patients with sexual dysfunction concluding that there is a significant association between ED and cardiovascular parasympathetic impairment. In a highly selected group of patients with no clinically significant cardiovascular disease, is ED still always associated with CAN? It has been proved that vascular ED could help in detection of heart disease before development of symptoms (Gazzaruso et al., 2004), but can ED secondary to autonomic failure predict future development of CAN?

In our study, we assessed this hypothesis in a specific group of males with type 2 diabetes with highly selected characteristics. Patients in our cohort had no evidence of 
ischaemic heart disease, hypertension, renal, neurological or thyroid disease.

\section{Patients and methods}

In our study, males with type 2 diabetes were recruited from Diabetes Clinic, St Lukes Hospital, Malta. In the first stage, each consecutive patient attending the clinic on each successive Wednesday, who had never complained of any significant shortness of breath on exertion or at rest, paroxysmal nocturnal dyspnoea, orthopnoea or lower limb swelling, chest pain, loss of consciousness or palpitations were chosen to enter the study. Any of the patients chosen, who had any of the following criteria was excluded:

1. Those on drugs known to affect autonomic nervous system, e.g. anti hypertensives, phenothiazines, tricyclic anti-depressants, benzodiazepines.

2. Those with overt renal failure, thyroid disease and those with a history or clinical evidence of cardiovascular or neurological disease or any other systematic disease.

3. Patients with a history of hypertension.

4. Those with any disease or condition causing polyneuropathy.

Those who fulfilled these criteria had their blood pressure checked after $5 \mathrm{~min}$ of rest on at least two occasions. The mean was taken. Those with a blood pressure of $>130 / 80$ were excluded from the study. The blood pressure was rechecked at each different stage of the study. Initially, 38 patients were recruited over 4 months.

On entry into the study patients were identified by an entry number. The data collected for every patient included their age, year of diagnosis of diabetes, antidiabetic treatment and history of smoking. Their glycosylated haemoglobin (Hba1C) level was also measured.

Informed consent was obtained from all subjects. This study was approved by the ethics committee of the University of Malta.

A maximal ECG exercise stress test using the standard Bruce protocol was carried out on all 38 patients, with a threadmill using a 12-lead ECG (Marquette CASE 8000 exercise testing system). Each patient as a minimum needed to attain $90 \%$ age-predicted maximum heart rate for the test to be valid. Standard features for positivity were used, namely: ischaemic chest pain consistent with angina during exercise, a $10 \mathrm{mmHg}$ or more drop in systolic BP during exercise when compared to baseline, exercise induced arrhythmias and planar or downsloping ST depression of $1 \mathrm{mV}$ or more at $80 \mathrm{~ms}$ from J-point. Those patients who had a positive stress test were excluded from the study and were referred for a coronary angiogram. Those with an abnormal resting ECG or anyone developing significant shortness of breath or intermit- tent claudication were also excluded. Ten patients in total were eliminated.

To exclude other potential causes for ED besides autonomic neuropathy, each of the other 28 patients had a Hospital Anxiety and Depression Scale test to exclude psychological disease (Zigmond \& Snaith, 1983), had their peripheral lower limb pulses examined, were assessed for loss of perianal sensation and their blood testosterone and prolactin levels measured. Any abnormalities in these tests excluded the patient from the study. Those drinking more than three units of alcohol every day or those with a history of prostate problems or pelvic pathology were also excluded. Four patients were excluded.

During the period of the first stage of the study, further two patients were excluded after being started on ACE inhibitors by their GP for development of hypertension. Thereafter 22 patients were assessed for CAN and ED in the second stage of the study.

\section{Erectile dysfunction assessment}

Each patient who entered the second stage was assessed for ED. The assessment was made by using the International Index of Erectile Function-5 (IIEF-5) as a diagnostic tool (Rosen et al., 1999). The questions asked were the following: Over the past 6 months: (i) How do you rate your confidence that you could get and keep an erection? (ii) When you had an erection with sexual stimulation, how often were your erections hard enough for penetration? (iii) During sexual intercourse, how often were you able to maintain your erection after you had penetrated your partner? (iv) During sexual intercourse, how difficult was it to maintain your erection to completion of intercourse? and (v) When you attempted sexual intercourse, how often was it satisfactory for you? The IIEF-5 score represents the sum of these questions with a maximum score of 25; patients with a score of 21 or less were taken to be suffering from ED. The questionnaires were analysed in a blinded fashion independently of the results of CAN testing.

\section{Cardiovascular autonomic neuropathy assessment}

Each patient then underwent tests for CAN. The three tests of cardiovascular autonomic nerve function were: (i) the Expiration : Inspiration (E: I) ratio (obtained from $\mathrm{R}-\mathrm{R}$ variations); (ii) the Valsalva ratio; and (iii) the standing $30: 15$ ratio. These tests use deep breathing, the Valsalva manoeuvre, and standing from a supine position, respectively, as provocative stimuli. Autonomic tests were applied under standardized conditions between 09:00 and 12:00 $\mathrm{AM}$, at least $2 \mathrm{~h}$ after following a light breakfast. 
The patients were not allowed to drink coffee or tea or smoke on the day of the testing. Diagnosis of CAN was based on an abnormality in one of the tests assessing heart rate variability (HRV); this was confirmed on repeating the test a week after. It has been stated that an abnormality in one of the above tests of HRV is indicative of early autonomic neuropathy (Vinik et al., 2003).

\section{E : I ratio}

The subject remained supine and took breaths deeply at the rate of one breath per $10 \mathrm{~s}$ (i.e. six breaths per minute) for $1 \mathrm{~min}$ while being monitored by ECG. The E : I is the ratio of the mean of the longest R-R intervals during deep expirations to the mean of the shortest $\mathrm{R}-\mathrm{R}$ intervals during deep inspirations. This is an indication of parasympathetic function. The following age-related values were used to define abnormal/normal levels: lowest normal value of E/I ratio: age 20-24:1.17; 25-29: 1.15; $30-34: 1.13 ; \quad 35-30: 1.12 ; \quad 40-44: 1.10 ; \quad 45-49: 1.08$; 50-54:1.07; 55-59:1.06; 60-64:1.04; 65-69: 1.03; 70-75 : 1.02 (Vinik, 2002; Vinik \& Mehrabyan, 2003).

\section{Valsalva manoeuvre}

In the standard Valsalva manoeuvre, the supine patient was connected to an ECG monitor, and forcibly exhaled for $15 \mathrm{~s}$ against a fixed resistance with an open glottis. The patient maintained constant pressure at $40 \mathrm{ml}$ over the 15-s interval. The Valsalva ratio is the longest $\mathrm{R}-\mathrm{R}$ occurring within $45 \mathrm{~s}$ of peak heart rate divided by the shortest R-R and is indicative of overall condition of the parasympathetic and sympathetic fibres (mainly parasympathetic). Ratios $\leq 1.20$ were taken as abnormal while those $\geq 1.21$ were normal (Ewing et al., 1973).

\section{Heart rate response to standing}

The patient was connected to the heart rate monitor while in the supine position. The patient then stood up to a full upright position, and the ECG was monitored for an additional period while standing. The heart rate tracing was used to calculate the ratio of the longest R-R interval (about beat 30) after the stand to the shortest $\mathrm{R}-\mathrm{R}$ interval (about beat 15). This measure, called the $30: 15$ ratio, reflects the overall condition of the para- sympathetic fibres. Ratios $\leq 1.030$ were taken as abnormal while those $\geq 1.031$ were normal (Campbell et al., 1978).

\section{Statistical methods}

Frequencies of impotence and CAN in the sample are established. The results of the cardiovascular tests are expressed as mean and 95\% confidence intervals (CI) for this small sample. As the sample size was small, statistical analysis was carried out using nonparametric tests such as the Fisher's exact test and the Mann-Whitney test. Logistic regression analysis was used to assess for confounding factors. A result was considered significant when $P$ was $<0.05$.

\section{Results}

The mean age of the patients reaching the second stage was 54.62 (95\% CI 50.63-58.6 years) and the mean resting heart rate was $83 \mathrm{bpm}$ (95\% CI 77-88). Age and heart rate are two potential confounding factors when assessing CAN. The patient characteristics for the two main groups and $P$-values for the differences between groups are tabulated (Table 1).

From this sample of 22 patients, 10 patients were found to have ED, that is attained a score of 21 or less on IIEF-5, whilst six patients were suffering from autonomic neuropathy, using criteria specified above.

From the 12 patients with no ED, three patients were suffering from CAN, whilst from the 10 patients with ED, three patients had CAN. There was no significant association $(P=1.00)$ between autonomic ED and CAN in this cohort. This analysis was performed using the Fisher's exact test. Figure 1 shows the graphical representation of these findings.

When assessing $\mathrm{E}: \mathrm{I}$ ratios, one of 10 patients (10\%) with ED had an impaired response whilst one of 12 patients $(8.3 \%)$ without ED had evidence of CAN. The mean value for the $\mathrm{E}$ : I ratios (difference of measured $\mathrm{E}$ : I ratios from normal target values for each patient's age) in the ED group was 0.147 (95\% CI $0.055-0.24$ ) whilst that in the non-ED group was 0.141 (95\% CI $0.034-0.21)$. This difference was not significant $(P=0.64)$ (Fig. 2).

Table 1 Patient data (mean values and 95\% confidence intervals)

\begin{tabular}{|c|c|c|c|c|}
\hline & $\begin{array}{l}\text { Age (years) } \\
(P=0.021)\end{array}$ & $\begin{array}{l}\text { Diabetes duration since } \\
\text { diagnosis (years) }(P=0.183)\end{array}$ & $\begin{array}{l}\text { Hba1c }(\%) \\
(P=0.972)\end{array}$ & $\begin{array}{l}\text { Resting heart rate } \\
(\mathrm{bpm})(P=0.791)\end{array}$ \\
\hline Erectile dysfunction & $59.7(54.5-64.7)$ & $10.1(4.1-16.0)$ & $7.4(5.6-9.3)$ & $83.4(74.8-92.1)$ \\
\hline No erectile dysfunction & $50.8(44.6-56.9)$ & $6.0(4.2-7.7)$ & $7.1(5.7-8.5)$ & $82.3(71.3-93.3)$ \\
\hline
\end{tabular}

Hba1c, glycosylated haemoglobin. 


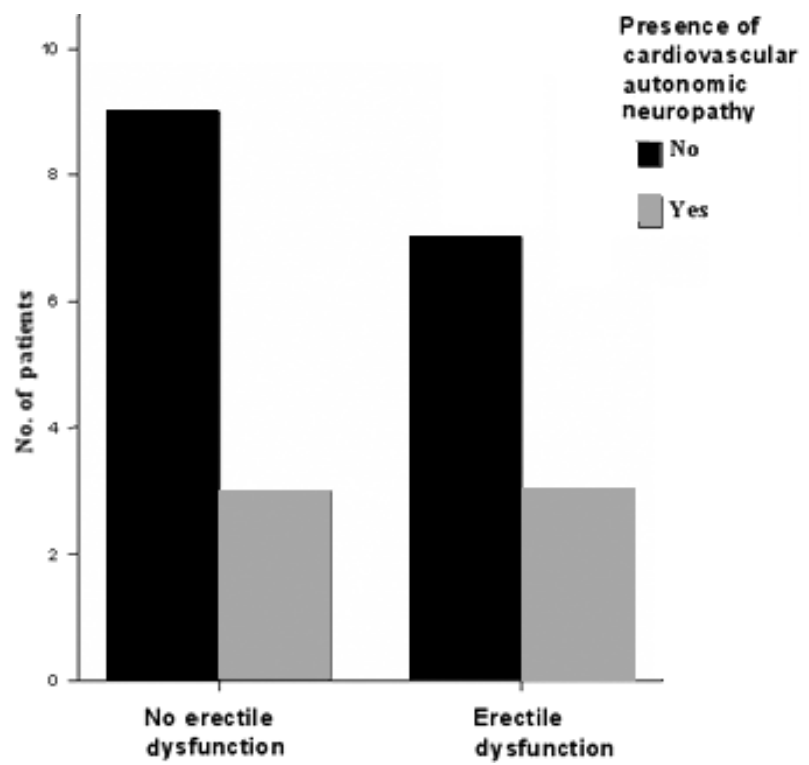

Fig. 1 Presence of erectile dysfunction and cardiovascular autonomic neuropathy in study group.

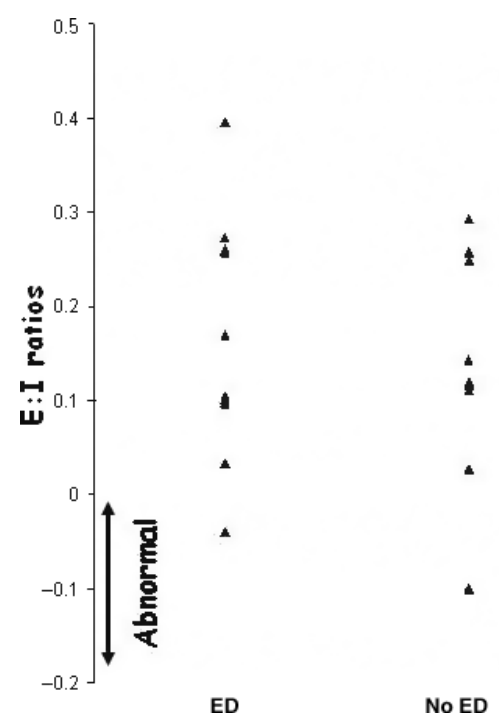

Fig. 2 Calculated difference in expiratory: inspiratory ratios from normal target values in patients with or without erectile dysfunction (ED).

When assessing Valsava ratios two of 10 patients (20\%) with ED had an abnormal response whilst three of 12 patients $(25 \%)$ not suffering from ED had impaired ratios. The mean value for Valsava ratios was 1.44 (95\% CI 1.27-1.61) in the ED group with mean value for the non-ED group being 1.45 (95\% CI 1.29-1.55). The difference was again not significant $(P=0.84)$ (Fig. 3 ).

None of the patients in the sample had an abnormal 30 : 15 ratio test. The mean value for the ED group was

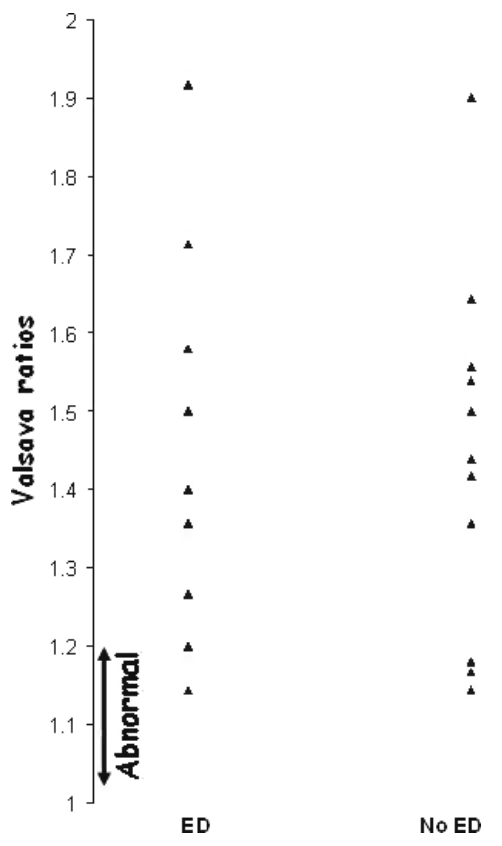

Fig. 3 Valsava ratio measurements in patients with or without erectile dysfunction (ED).

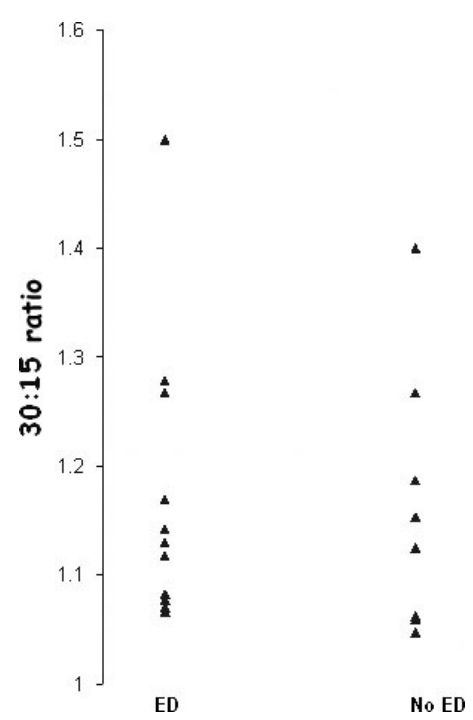

Fig. 4 30:15 ratio test in patients with or without erectile dysfunction (ED).

1.162 (95\% CI 1.08-1.24) and for the non-ED group was 1.168 (95\% CI 1.05-1.27) with a nonsignificant difference between the groups $(P=0.947)$ (Fig. 4).

Through logistic regression analysis, for each $1 \%$ rise in Hbalc there was no significant increase in association between ED and CAN $(P=0.866)$. There was also no significant increase in association between CAN and ED when adjusting for the time from diagnosis $(P=0.097)$ 
although in this case a larger sample might have resulted in a significant end result. On the other hand, age was found to have a significant effect; the association between CAN and ED significantly increased $(P=0.036)$ with age. In the study, five of 12 patients $(41.7 \%)$ with no ED were ex-smokers whilst three of 10 patients (30\%) with ED smoked. Using the Mantel-Haenszel Common odds ratio estimation, smoking did not significantly effect the association between CAN and ED $(P=0.907)$.

\section{Discussion}

Reduced HRV is the earliest indicator of CAN. CAN has a prevalence of between $17 \%$ and $22 \%$ in patients with type 1 and type 2 diabetes (Neil et al., 1989). The importance of CAN with respect to morbidity and mortality for patients with diabetes has led to various guidelines being developed for assessment of autonomic dysfunction. According to the American Academy of Neurology (1996) the tests used in our study are established, and are accepted as appropriate by medical practitioners, when used for testing for CAN. Vinik et al. (2003) state that an abnormal result in one test for HRV is indicative of autonomic neuropathy.

In our study, when using these criteria, we found that there was no significant association between the presence of autonomic ED and CAN in a well characterised group $(P=1)$. Each different test for CAN did not show a significant association. The strength of our study lies in the fact that our patients were likely to be suffering from ED secondary to diabetic autonomic neuropathy and not to any other cause. Great care was taken to exclude patients with hypertension or overt cardiovascular disease, endocrine, and other neurological or psychological illnesses, all of which would be confounding variables for a cause for ED. Besides this, our patients did not abuse alcohol and had no history of prostate disease or other pelvic pathology. To our knowledge, no other study has evaluated this association in this way. Quadri et al. (1989) revealed an association between E : I HRV tests and impotence, however, they did not characterize ED similarly.

Our study reveals that ED is not a reliable diagnostic marker of CAN, but it brings up another important point. Ewing et al. (1980) had revealed that in a group of patients with diabetes suffering from impotence alone, these developed abnormal tests for CAN in the follow-up period. In our analysis, we notice that with age the association between CAN and ED significantly increases $(P=0.036)$. Besides this, there could possibly be an association between the years from diagnosis of diabetes and the development of CAN in patients with ED $(P=0.097)$. If a larger sample was studied, this might have been significant. Therefore, these findings should alert us that ED may be an early clinical indicator of forthcoming autonomic damage.

One of the main limiting factors for the study is that the sample size is small. One must understand that patients with diabetes with these special characteristics are not easy to recruit and a substantial amount of patients were lost in the stringent exclusion criteria used. The very high nonsignificant $P$-value of 1.00 when assessing the association between CAN and ED indicates that increasing the sample size might not change the result. This makes our results valid. Age was significantly different between groups. This observation was expected as the incidence of ED has been shown to be strongly associated with age (Keles et al., 2005). The only way to eliminate this confounding factor would be to select an age-matched group, but this would restrict further an already limited sample population. As there was no significant association between ED and CAN in this cohort, this finding has no influence on the outcome of the study. Another limitation is that to exclude a vascular cause for ED we did not directly measure the penile blood pressures. Chiurlia et al. (2005) revealed that coronary atherosclerosis is more severe in patients with vascular ED. Keeping these findings in mind, to eliminate patients with a possibility of vascular ED, we excluded patients with a high probability of cardiovascular disease, that is those with history of coronary artery disease, exertional chest pain, positive elective stress tests, hypertension, intermittent claudication and nonpalpable lower limb pulses. Caused by these limitations our investigation should be primarily considered as a pilot study.

To conclude, this study, although small, demonstrates that ED in patients with type 2 diabetes, with no associated comorbidities, is not diagnostic of CAN. Most importantly, this study reveals that the presence of ED might be a portent of future CAN. We therefore insist that at least simple bedside tests for autonomic neuropathy should be performed routinely on patients with diabetes suffering from ED. This will help establish the diagnosis of CAN as early as possible and thus prevent associated mortality and morbidity. The results of this study support the need for further research, through larger experimental designs, in this area.

\section{References}

American Academy of Neurology (1996) Clinical autonomic testing report of the Therapeutics and Technology Assessment Subcommittee of the American Academy of Neurology: assessment. Neurology 46:873-880.

Bacon CG, Hu FB, Giovannucci E, Glasser DB, Mittleman MA, Rimm EB (2002) Association of type and duration of diabetes with erectile dysfunction in a large cohort of men. Diabetes Care 25:1458-1463. 
Campbell IW, Murray A, Neilson JM, Clarke BF (1978) Immediate heart response to standing: simple test for autonomic neuropathy in diabetes. BMJ 21:145-147.

Chiurlia D, Amico R, Ratti C, Granata A, Romagnoli R, Modena M (2005) Subclinical coronary artery atherosclerosis in patients with erectile dysfunction. J Am Coll Cardiol 46:1503-1506.

Ewing DJ, Campbell IW, Burt AA, Clarke BF (1973) Vascular reflexes in diabetic autonomic neuropathy. Lancet 2: 1354-1356.

Ewing DJ, Campbell IW, Clarke BF (1980) The natural history of diabetic autonomic neuropathy. Q J Med 49:95-108.

Gazzaruso C, Giordanetti S, De Amici E, Bertone G, Falcone C, Geroldi D, Fratino P, Solerte SB, Garzaniti A (2004) Relationship between erectile dysfunction and silent myocardial ischemia in apparently uncomplicated type 2 diabetic patients. Circulation 110:22-26.

Keles I, Aydin G, Orkun S, Basar MM, Batislam E (2005) Two clinical problems in elderly men: osteoporosis and erectile dysfunction. Arch Androl 51:177-184.

Neil HAW, Thompson AV, John S, McCarthy ST, Mann JI (1989) Diabetic autonomic neuropathy: the prevalence of impaired heart rate variability in a geographically defined population. Diabet Med 6:20-24.

Pfeifer MA, Weinberg CR, Cook DL, Reenan A, Halter JB, Ensick JW, Porte D (1984) Autonomic neural dysfunction in recently diagnosed diabetic subjects. Diabetes Care 7:447453.

Quadri R, Veglio M, Flecchia D, Tonda L, De Lorenzo F, Chiandussi L, Fonzo D (1989) Autonomic neuropathy and sexual impotence in diabetic patients: analysis of cardiovascular reflexes. Andrologia 21:346-352.

Rosen RC, Cappelleri JC, Smith MD, Lipsky J, Peña BM (1999) Development and evaluation of an abridged, 5-item version of the international index of erectile function (IIEF5 ) as a diagnostic tool for erectile dysfunction. Int J Impot Res 11:319-326.

Vinik AI (2002) Diagnosing diabetic autonomic neuropathy. Medscape Diabetes Endocrinol 4. Available at: http://www. medscape.com/viewarticle/445092 (Accessed December 2006).

Vinik AI, Mehrabyan A (2003) Diagnosis and management of diabetic autonomic neuropathy. Compr Ther 29:130-145.

Vinik AI, Maser RE, Mitchell BD, Freeman R (2003) Diabetic autonomic neuropathy. Diabetes Care 26:1553-1579.

Ziegler D (1999) Cardiovascular autonomic neuropathy: clinical manifestations and measurement. Diabetes Rev 7:300-315.

Ziegler D (2001) Diagnosis and treatment of diabetic autonomic neuropathy. Curr Diab Rep 1:216-227.

Zigmond AS, Snaith RP (1983) The Hospital Anxiety and Depression Scale. Acta Psychiatr Scand 67:361-370. 